Research Article

\title{
Dominating Number on Icosahedral-Hexagonal Network
}

\author{
Miroslava Mihajlov Carević $(\mathbb{C}$ \\ Faculty of Mathematics and Computer Science, Alfa BK University, Belgrade, Serbia \\ Correspondence should be addressed to Miroslava Mihajlov Carević; miroslava.carevic.mihajlov@alfa.edu.rs
}

Received 12 December 2020; Revised 23 March 2021; Accepted 26 March 2021; Published 12 April 2021

Academic Editor: Fazal M. Mahomed

Copyright @ 2021 Miroslava Mihajlov Carević. This is an open access article distributed under the Creative Commons Attribution License, which permits unrestricted use, distribution, and reproduction in any medium, provided the original work is properly cited.

In this paper, we deal with the dominating set and the domination number on an icosahedral-hexagonal network. We will consider all cases of successive halving of the edges of triangles that are the sides of icosahedrons and thus obtain icosahedralhexagonal networks.

\section{Introduction}

The German weather service has developed an operational global numerical model for weather forecasting, called GME, based on the icosahedral-hexagonal network. In this paper [1], the authors describe this network and provide estimates of the accuracy of its application. In order to generate a network in the sphere, a regular icosahedron was constructed (Figure 1) so that 2 of the 12 verticals passing through the 12 vertices of the icosahedron coincide with the north and south poles.

By successive halving of the edges of the triangles that are the sides of the icosahedron, new triangles are formed (Figure 2). The parameter $n$ represents the number interval on the starting triangle of the icosahedral network.

The icosahedral network develops into a hexagonal network consisting of 10 rhombuses, each of which is composed of 2 triangles from the icosahedral network (Figure 3).

The icosahedral-hexagonal network was first introduced into meteorological modeling in 1968. In recent years, there has been a growing interest of researchers in this type of network.

In the previous period, the icosahedral network was the subject of analysis by the author of this paper. In paper [2], the generating function for icosahedral figurative numbers is constructed.

In paper [3], the results of our research on the dominating set and domination number of an icosahedral-hexagonal network without halving the edges of the triangles that are the sides of an icosahedron are presented.

In this paper, we consider an icosahedral-hexagonal network with successive halving of the edges of the triangles and determine domination numbers for the network thus obtained.

\section{Materials and Methods}

Like usual in mathematics, we denote the vertex set and edge set of the graph $G$ by $V(G)$ and $E(G)$, respectively.

A subset $D$ of $V(G)$ is called a $t$-dominating set, if for every vertex $y$ not in $D$, there exists at least one vertex $x$ in $D$, such that $d(x, y) \leq t$. With $d(x, y)$, we denote distance between the vertices $x$ and $y$.

The number of elements of the smallest $t$-dominating set is called the $t$-domination number and is denoted by $\gamma_{t}$. For $t=1$, 1-dominating set is called dominating set and 1-domination number is also called domination number and denoted by $\gamma$.

In the previous period, $t$-dominance was investigated on hexagonal [4-7], rectangular [8], and triangular [9] cactus 


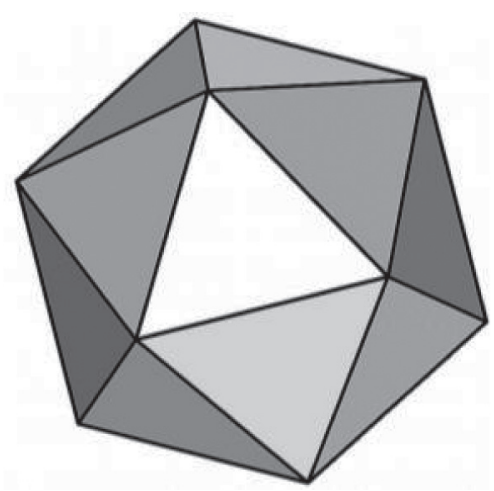

FIGURE 1: Regular icosahedron (the image is taken from paper [1]).
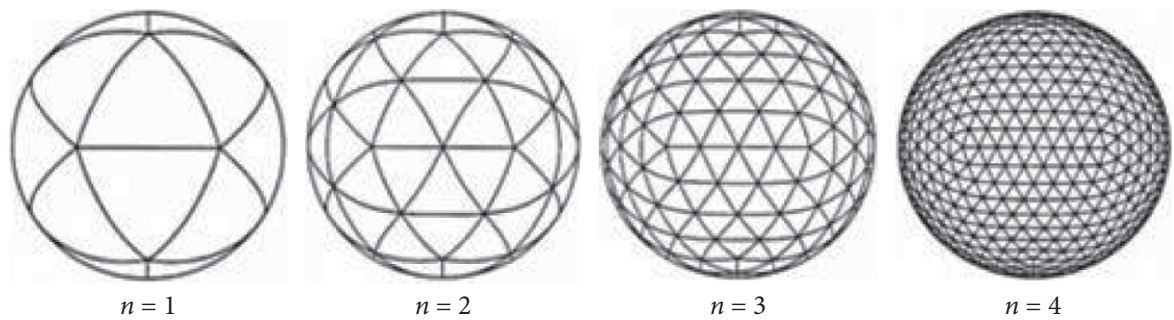

Figure 2: Hunting the edges of the icosahedron (the image is taken from paper [1]).

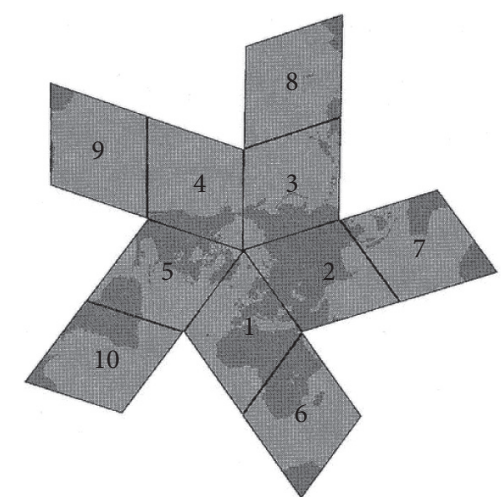

FIgURE 3: Icosahedral-hexagonal network GME (the image is taken from paper [1]).

chains. Research was also conducted for $R$-domination in graphs [10], $k$-dominating number of Cartesian products of two paths [11], paid domination in graphs [12], and total and double-total domination number on the hexagonal network [13].

\section{Results and Discussion}

In this section, we are going to consider 1-domination (or only domination) on rhomboidal networks composed of triangles that are sides of an icosahedron. We are going to analyze all cases of successive halving of the edges of triangles on $n=2^{k}$ parts where $k \in N$.

We denoted the rhomboidal-triangular network with $m$ rows and $c$ columns with $R_{m, c}$ and with $R_{m c}$ vertices in this network. The initial rhomboidal-triangular network consists of 2 rhombuses, i.e., 4 triangles from the icosahedral- hexagonal network (Figure 4). The complete icosahedralhexagonal network consists of 5 rhomboidal-triangular networks.

In paper [3], it was proved that the 1-domination number for $R_{2,3}$ is equal to 2 . By halving the edges of the triangles in the rhomboidal-triangular network $R_{2}$, 3, we obtain different cases that are the subject of the analysis in this paper. We denote the number of divisions of the triangles edges in the network $R_{2,3}$ by $n$.

Lemma 1. For $n=2$, the domination number is $\gamma\left(R_{3,5}\right)=3$.

Proof. By dividing the edges of the triangle in the network $R_{2,3}$ into 2 parts, we obtain the network $R_{3,5}$ (Figure 5).

Let us prove that the minimum dominating set for $R_{3,5}$ is the set $D_{1}=\left\{R_{21}, R_{23}, R_{25}\right\}$. 


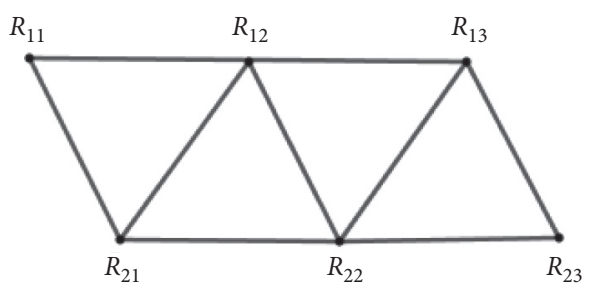

Figure 4: Rhomboidal-triangular network $R_{2,3}$.

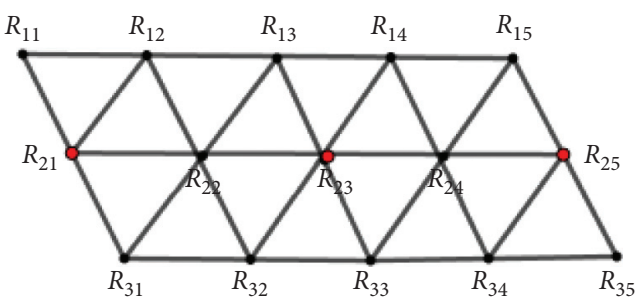

FIgURE 5: Rhomboidal-triangular network $R_{3,5}$.

Node $R_{21}$ dominates nodes $R_{11}, R_{12}, R_{22}$, and $R_{31}$. Node $R_{23}$ dominates nodes $R_{13}, R_{14}, R_{22}, R_{24}, R_{32}$, and $R_{33}$. Node $R_{25}$ dominates nodes $R_{15}, R_{24}, R_{34}$, and $R_{35}$. Hence, these 3 nodes dominate all nodes in the network $R_{3}, 5$. Suppose that the set $D_{1}$ is not a minimal dominating set but that there is a set $D_{1}^{\prime}$ of less cardinality that dominates the network $R_{3,5}$. The set $D_{1}^{\prime}$ would then have 2 nodes that would dominate over the 13 remaining nodes in the network $R_{3,5}$. One node $R_{i j}$ in the grid can dominate a maximum of 6 nodes of the hexagon in the center of which is the node $R_{i j}$. Hence, two nodes can dominate a maximum of 12 nodes. These would be nodes $R_{22}$ and $R_{24}$. However, they cannot dominate over 13 nodes (they are not dominant for nodes $R_{11}$ and $R_{35}$ ). Hence, the set $D_{1}{ }^{\prime}=\left\{R_{22}, R_{24}\right\}$ is not dominant for the network $R_{3,5}$. Similarly, it is proved that no other set of 2 nodes can dominate the entire network $R_{3,5}$. We can conclude that $D_{1}$ is the minimum dominating set for the network $R_{3,5}$ and its cardinality is 3 , therefore $\gamma\left(R_{3,5}\right)=3$.
Lemma 2. For $n=2^{2}$, the domination number is $\gamma\left(R_{5,9}\right)=8$.

Proof. By dividing the edges of the triangle in the network $R_{2,3}$ into 4 parts we obtain the network $R_{5,9}$ (Figure 6).

Let us look at the set $D_{2}=\left\{R_{14}, R_{21}, R_{26}, R_{28}, R_{42}, R_{44}, R_{49}\right.$, $\left.R_{56}\right\}$. Nodes $R_{42}$ and $R_{44}$ dominate nodes $R_{3 i}$ and $R_{5 j}$ for $i=2$, 3 , 4, and 5 and $j=1,2,3$, and 4 . Node $R_{21}$ dominates nodes $R_{11}, R_{12}, R_{22}$, and $R_{31}$. Node $R_{14}$ dominates nodes $R_{13}, R_{15}$, $R_{23}$, and $R_{24}$. Similarly, nodes $R_{26}, R_{28}, R_{56}$, and $R_{49}$ dominate the nodes in the second rhombus including nodes $R_{25}$ and $R_{55}$ that were not covered by the previous dominance. So the set $D_{2}$ dominates all nodes in the network $R_{5,9}$.

Let us prove that $D_{2}$ is the minimal set that dominates over all nodes in the network $R_{5,9}$. Suppose there is a set $D_{2}$ ' whose cardinality is less than 8 (supposing 7) and which dominates the network $R_{5,9}$. As mentioned earlier, one node in a network can dominate a maximum of 6 nodes that form a hexagon around it. Note that the network $R_{5,9}$ is composed of 8 hexagons and 2 more nodes $R_{11}$ and $R_{59}$. The seven adjacent hexagons in the network have common nodes over which they dominate. The total number of nodes without repeating dominance for 7 hexagons in the grid is $31(6+3$. $5+4+2 \cdot 3)$. If we add to this number the number of nodes that dominate them (7), we get the sum of 38 . As the network $R_{5,9}$ has 45 nodes, there are still 7 uncovered nodes, which leads us to the conclusion that there is no set $D_{2}{ }^{\prime}$ whose cardinality is less than 8 and which dominates the network $R_{5,9}$. Hence, $D_{2}$ is the minimum dominating set for the network $R_{5,9}$ and its cardinality is 8 , therefore $\gamma\left(R_{5,9}\right)=$ 8.

Lemma 3. For $n=2^{3}$, the domination number is $\gamma\left(R_{9,17}\right)=$ 32 .

Proof. By dividing the edges of the triangle in the network $R_{2,3}$ into 8 parts, we get the network $R_{9,17}$ (Figure 7).

Observe that the network $R_{9,17}$ is composed of 4 networks $R_{5,9}$. By applying the results expressed in Lemma 2, we form a set:

$$
\begin{aligned}
D_{3}= & \left\{R_{1 i}, i=4,12\right\} \cup\left\{R_{2 j}, j=1,6,8,9,14,16\right\} \cup\left\{R_{4 k}, k=2,4,9,10,12,17\right\} \cup\left\{R_{5 m}, m=4,6,12,14\right\} \\
& \cup\left\{R_{6 n}, n=1,6,8,9,14,16\right\} \cup\left\{R_{8 p}, p=2,4,9,10,12,17\right\} \cup\left\{R_{9 q}, q=6,14\right\} .
\end{aligned}
$$

Based on the proof in Lemma 2 and the fact that the network $R_{9,17}$ is composed of 4 networks $R_{5,9}$, we conclude that the set $D_{3}$ dominates over all nodes in the network $R_{9,17}$. Suppose that there is a set $D_{3}{ }^{\prime}$ of less cardinality that dominates the network $R_{9,17}$. Let us take that $D_{3}^{\prime}=D_{3} /\left\{R_{66}\right\}$. Then, the distance of node $R_{76}$ from the nearest nodes in the network is equal to 2 . So there is a node in the network over which no node from the set $D_{3}$ ' dominates. We come to the same conclusion by dropping any node from the set $D_{3}$. Based on this, we conclude that $D_{3}$ is the minimum dominating set for the $R_{9,17}$ network. Since the cardinality of the set $D_{3}$ is equal to 32 , it follows that $\gamma\left(R_{9,17}\right)=32$.
Lemma 4. For $n=2^{k} i k \in N /\{1\}$, the dominating number is $\gamma$ $\left(R_{n+1,2 n+1}\right)=2^{2 k-1}$.

Proof. We derive the proof by using the principle of mathematical induction. For $k=2$, we obtain the statement expressed in Lemma 2 where it is also proved. Suppose that the statement holds for $k=m, m \in N^{\wedge} m>2$, i.e., $\gamma\left(R_{n+1}\right.$, $2 n+1)=2^{2 m-1}$ where $n=2^{m}$. Thus, we assumed it was $\gamma\left(R_{2^{m}+1,2^{m+1}+1}\right)=2^{2 m-1}$.

Let us prove that the statement holds for $k=m+1$ i.e., $\gamma$ $\left(R_{n+1}, 2 n+1\right)=2^{2 m+1}$ where $n=2^{m+1}$. We need to prove that $\gamma\left(R_{2^{m+1}+1,2^{m+2}+1}\right)=2^{2 m+1}$. 


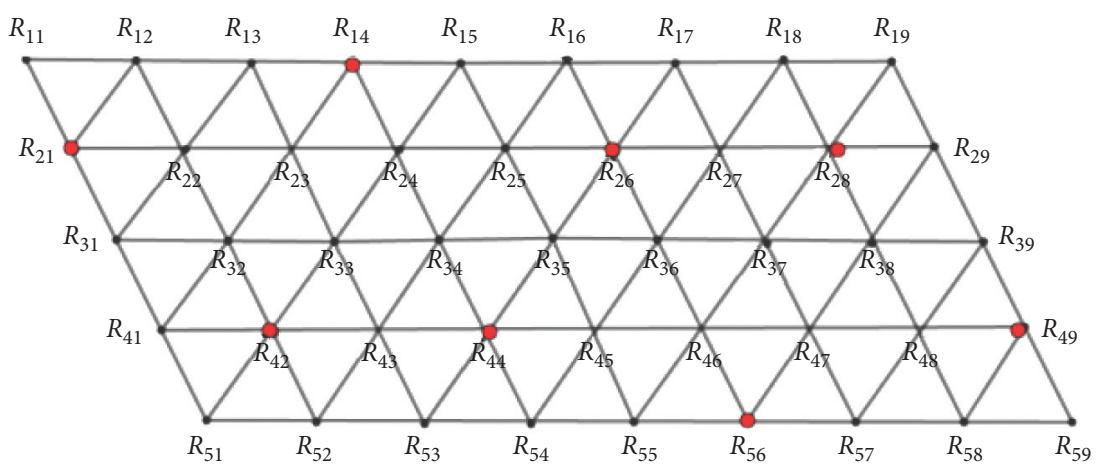

FIgURE 6: Rhomboidal-triangular network $R_{5,9}$.

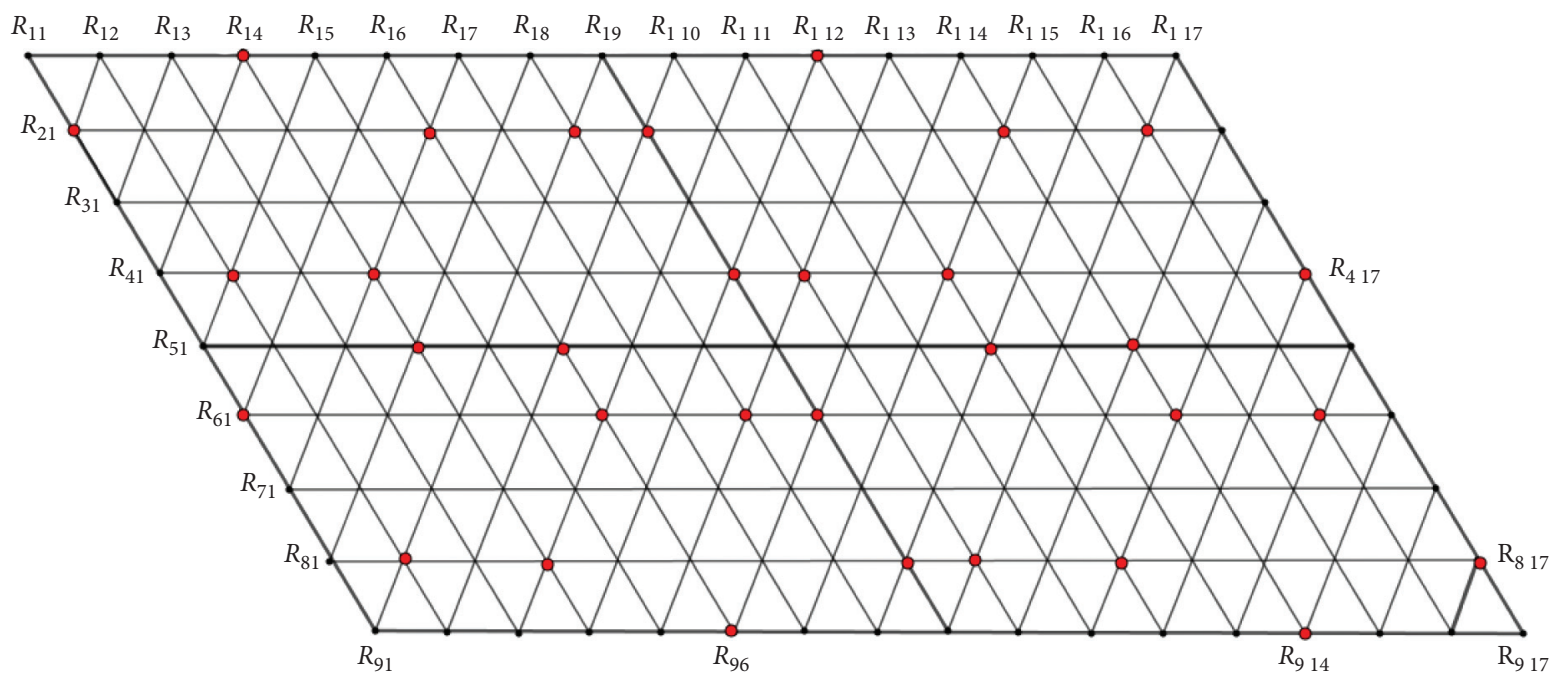

FIgURE 7: Rhomboidal-triangular network $R_{9,17}$.

For $k=m+1$, i.e., $n=2^{m+1}$, we obtain the division of the edges of the triangle in the initial network $R_{2,3}$ on $2^{m+1}$ parts and network $R_{2^{m+1}+1,2^{m+2}+1}$ which is composed of 4 networks $R_{2^{m}+1,2^{m+1}+1}$.

As it was proved in Lemma 3, it is not possible to remove any nodes from the dominating set for the network $R_{2^{m+1}+1,2^{m+2}+1}$ which we obtain by uniting 4 dominating sets for the network $R_{2^{m}+1,2^{m+1}+1}$ for $m \in N^{\wedge} m>2$. It follows that it is

$$
\begin{aligned}
\gamma\left(R_{2^{m+1}+1,2^{m+2}+1}\right) & =4 \cdot \gamma\left(R_{2^{m}+1,2^{m+1}+1}\right)=4 \cdot 2^{2 m-1} \\
& =2^{2} \cdot 2^{2 m-1}=2^{2 m+1},
\end{aligned}
$$

which needed to be proven.

\section{Main Results}

In this section, we deal with the dominance of the icosahedral-hexagonal network shown in Figure 8. This network is composed of 5 rhomboidal-triangular networks that we called "prongs" and are numbered from 1 to 5 as shown in Figure 8(a).
We marked the nodes in the icosahedral-hexagonal network with $R_{m c}^{p}$ where $p$ denotes the prong, $m$ denotes the rows, and $c$ denotes the column in the rhomboidal-triangular network of the prong $p$. The center of the icosahedralhexagonal network is the node denoted by $R_{1}$ (Figure $8(\mathrm{~b})$ ).

Notice that some nodes in the network match:

$$
\begin{aligned}
& R_{12}^{1} \equiv R_{21}^{2} \\
& R_{12}^{2} \equiv R_{21}^{3} \\
& R_{12}^{3} \equiv R_{21}^{4} \\
& R_{12}^{4} \equiv R_{21}^{5} i R_{12}^{5} \equiv R_{21}^{1} .
\end{aligned}
$$

As in the previous section, we are going to analyze all cases of successive halves of the edges of triangles of the initial network (Figure 8) on $n=2^{k}$ parts where $k \in N$. We denote the icosahedral-hexagonal network created by halving the edges of triangles into $n$ parts by $\mathrm{IH}_{n+1,2 n+1}$. In the next part, we deal with determining the domination number $\gamma\left(\mathrm{IH}_{n+1,2 n+1}\right)$ for the icosahedral-hexagonal network. 


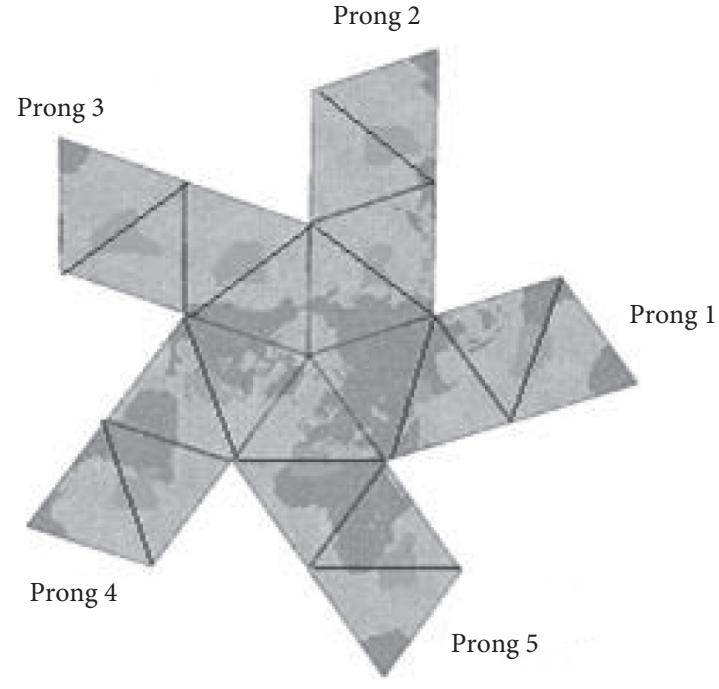

(a)

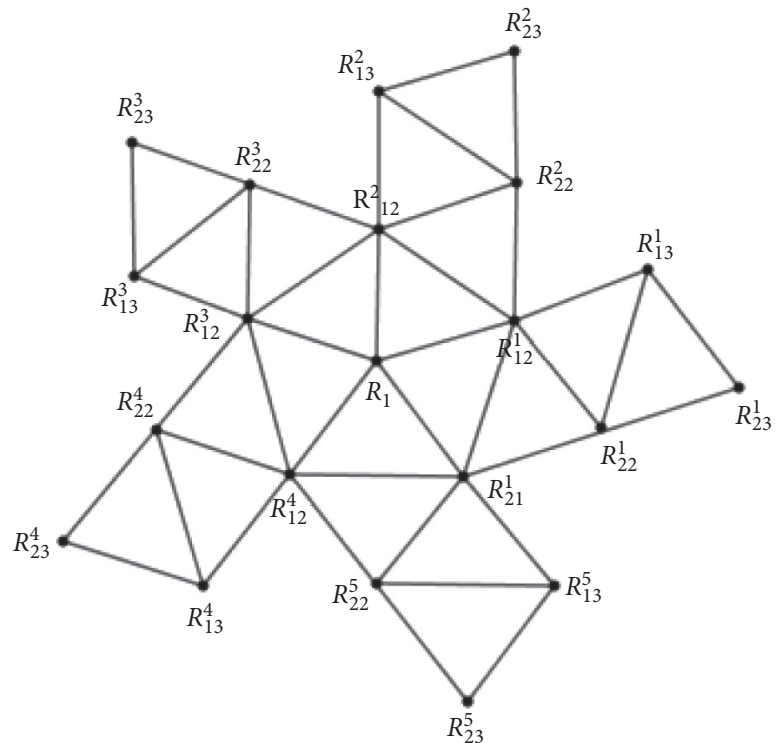

(b)

FIGURE 8: Icosahedral-hexagonal network with the prongs and indices.

Theorem 1. For $n=2$, the domination number for the icosahedral-hexagonal network is as follows:

$$
\gamma\left(I H_{3,5}\right)=11 \text {. }
$$

Proof. To prove this statement, we will use the results obtained in the previous section. By dividing the edges of the triangles in the icosahedral-hexagonal network shown in Figure 8 into 2 equal parts, we obtain the icosahedralhexagonal network shown in Figure 9.

The obtained icosahedral-hexagonal network is composed of 5 networks $R_{3,5}$ located in its prongs. In Lemma 1, it is proved that the dominating number for the network $R_{3,5}$ is equal to 3. Based on Lemma 1 , the set $D=\left\{R_{21}^{p}, R_{23}^{p}, R_{25}^{p}, p=1,2,3,4,5\right\}$ is the dominating set on the icosahedral-hexagonal network for $n=2$. But it is not the minimum dominating set. Node $R_{1}$ dominates over 5 nodes in its environment, and thus, it is possible to remove nodes $R_{21}^{1}, R_{21}^{2}, R_{21}^{3}, R_{21}^{4}$, and $R_{21}^{5}$. Hence, the minimum dominating set for this network is the set $D^{\prime}=\left\{R_{23}^{p}, R_{25}^{p}, p=\right.$ $1,2,3,4,5\} \cup\left\{R_{1}\right\}$. Its cardinality is 11 , so the dominance number for the icosahedral-hexagonal network is $\gamma\left(\mathrm{IH}_{3,5}\right)=$ 11 , which had to be proved.

Theorem 2. For $n=4$, the dominating number for the icosahedral-hexagonal network is as follows:

$$
\gamma\left(I H_{5,9}\right)=38 \text {. }
$$

Proof. By dividing the edges of the triangles in the icosahedral-hexagonal network $\mathrm{IH}_{2,3}$ into 4 equal parts, we obtain the icosahedral-hexagonal network $\mathrm{IH}_{5,9}$ shown in Figure 10.
The obtained icosahedral-hexagonal network is composed of 5 networks $R_{5,9}$ which are located in its prongs. In Lemma 2, it is proved that the domination number for the network $R_{5,9}$ is equal to 8 .

Based on Lemma 2, the set $D=\left\{R_{14}^{p}, R_{21}^{p}, R_{26}^{p}, R_{28}^{p}, R_{42}^{p}\right.$, $\left.R_{44}^{p}, R_{49}^{p}, R_{56}^{p}, p=1,2,3,4,5\right\}$ is the dominating set on the icosahedral-hexagonal network $\mathrm{IH}_{5}, 9$. But it is not a minimum dominating set. Node $R_{21}^{1} \equiv R_{12}^{5}$ can be removed because it dominates over nodes dominated by nodes $R_{12}^{1}, R_{41}^{1}$, and $R_{21}^{5}$. Also, node $R_{21}^{4} \equiv R_{21}^{3}$ can be removed because it dominates over nodes dominated by $R_{12}^{4}$ and $R_{41}^{4} i R_{21}^{3}$. No other node can be removed because some node in the network would be left without dominance. If we dropped the node $R_{12}^{2} \equiv R_{12}^{3}$, the node $R_{22}^{3}$ would remain without dominance. The same is true for node $R_{12}^{1} \equiv R_{12}^{2}$. So among the 5 nodes that make up the first circle around the central node $R_{1}$, it is possible to throw out only 2 nodes.

Let us observe other nodes in set $D$ that fulfill the condition that their mutual distance is equal to 1 . Such nodes have common nodes over which they dominate, so it is hypothetically possible to remove one of them. If we dropped the node $R_{41}^{4} \equiv R_{41}^{3}$, the nodes $R_{23}^{3}$ and $R_{24}^{3}$ would remain without dominance. If we dropped the node $R_{42}^{4}$, the nodes $R_{52}^{4}$ and $R_{33}^{4}$ would remain without dominance. The same is true for the other nodes in the set $D$. Based on that, the dominating set for the network $\mathrm{IH}_{5,9}$ is the set $D^{\prime}=D /\left\{R_{21}^{1}, R_{21}^{4}\right\}$.

Its cardinality is $5 \cdot 8-2$, i.e., 38 . Thus, $\gamma\left(\mathrm{IH}_{5,9}\right)=38$, which had to be proved.

Theorem 3. For $n=8$, the domination number for the icosahedral-hexagonal network is as follows: 


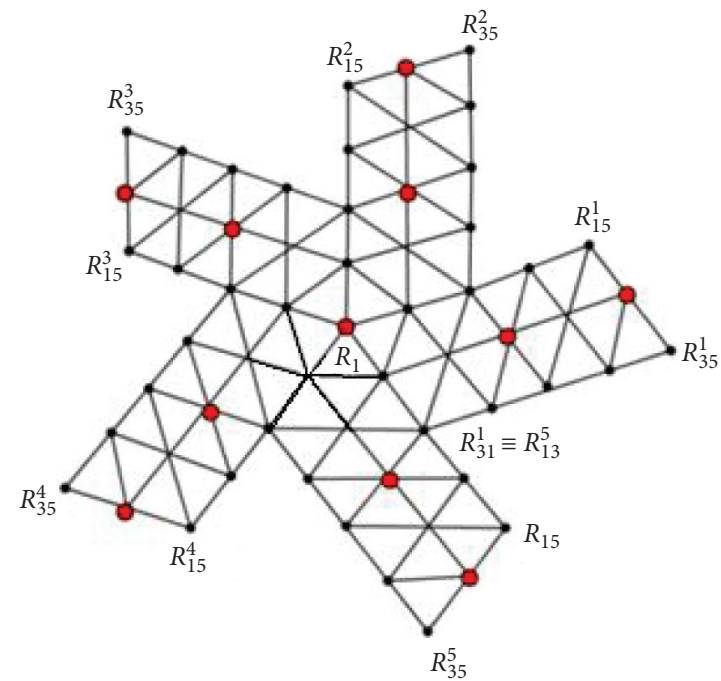

FIGURE 9: Icosahedral-hexagonal network $\mathrm{IH}_{3,5}$.

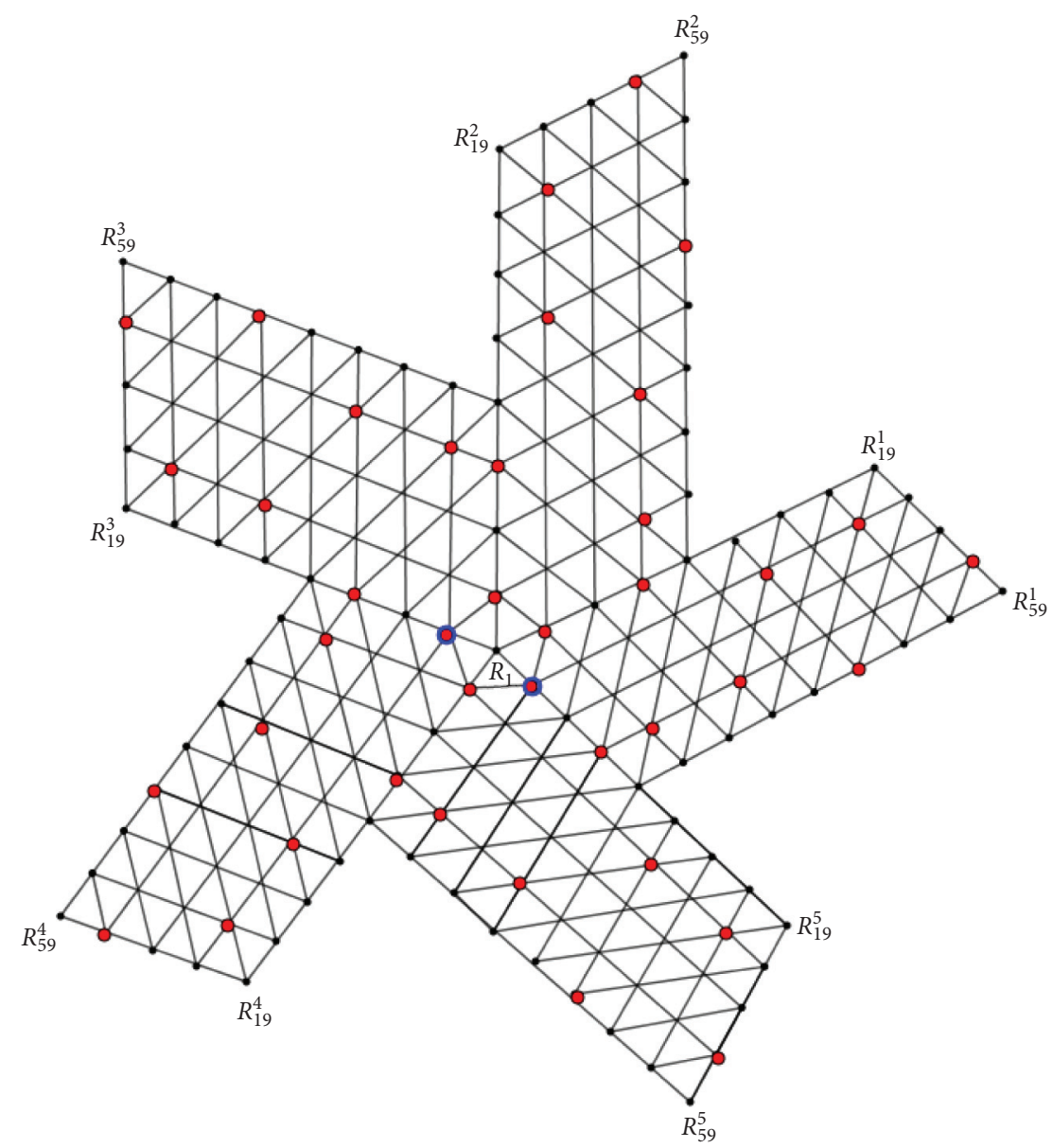

FIGURE 10: Icosahedral-hexagonal network $\mathrm{IH}_{5,9}$.

$\gamma\left(I H_{9,17}\right)=158$

(6)

Proof. By dividing the edges of triangles in an icosahedralhexagonal network $\mathrm{IH}_{2,3}$ by 8 equal parts, we obtain the icosahedral-hexagonal network $\mathrm{IH}_{9,17}$ shown in Figure 11 . 


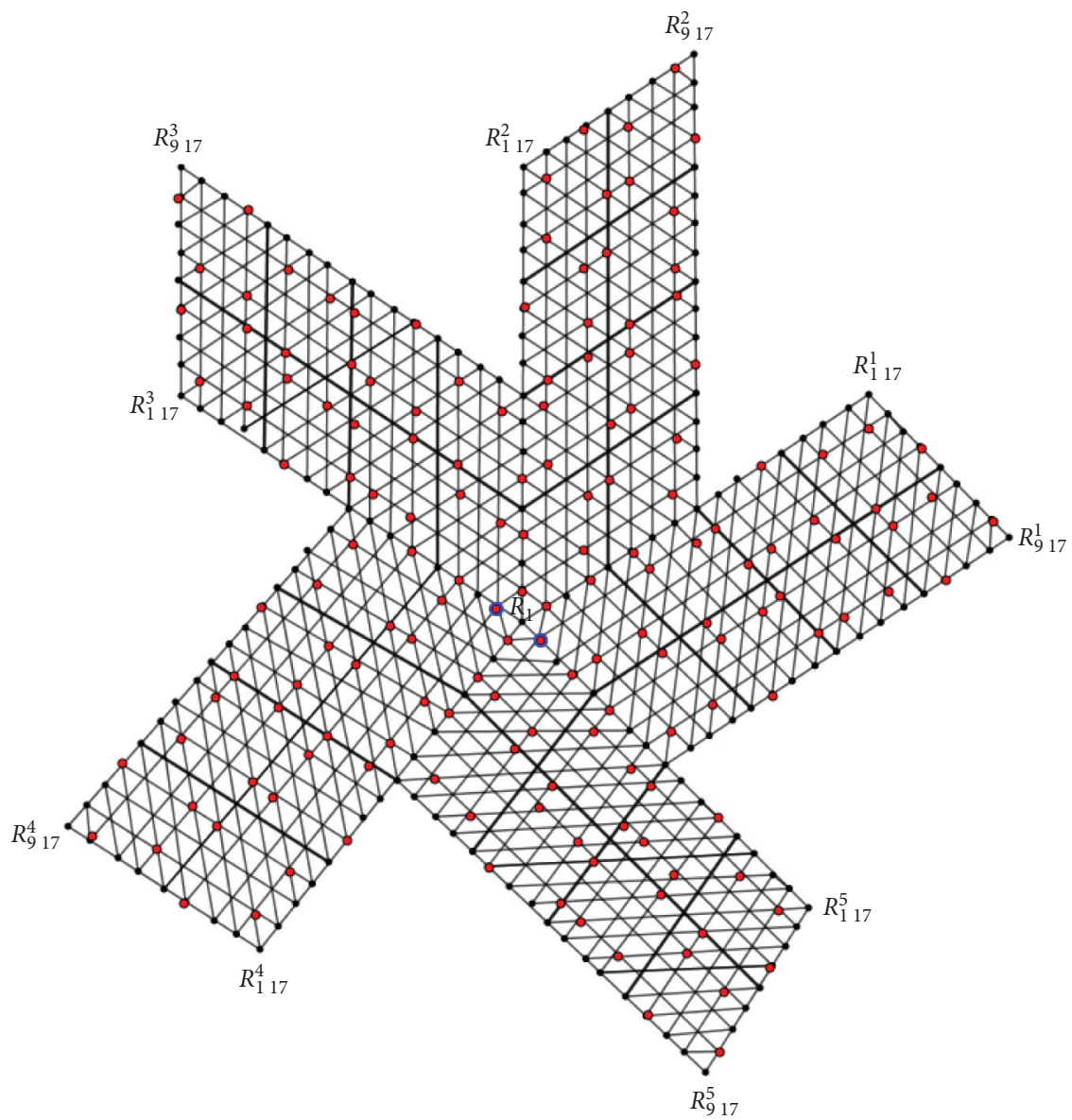

Figure 11: Icosahedral-hexagonal network $\mathrm{IH}_{9,}, 17$.

The obtained icosahedral-hexagonal network is composed of 5 networks $R_{9,17}$ which are located in its prongs. In
Lemma 3, it was proved that the domination number $\gamma$ $\left(R_{9,17}\right)=32$.

Based on Lemma 3 set,

$$
\begin{aligned}
D_{3}= & \left\{R_{1 i}^{p}, i=4,12\right\} \cup\left\{R_{2 j}^{p}, j=1,6,8,9,14,16\right\} \cup\left\{R_{4 k}^{p}, k=2,4,9,10,12,17\right\} \cup\left\{R_{5 m}^{p}, m=4,6,12,14\right\} \\
& \cup\left\{R_{6 n}^{p}, n=1,6,8,9,14,16\right\} \cup\left\{R_{8 p}^{p}, q=2,4,9,10,12,17\right\} \cup\left\{R_{9 q}^{s}, s=6,14\right\} z a, \quad p=1,2,3,4, \text { and } 5
\end{aligned}
$$

which is the dominating set on the icosahedral-hexagonal network $\mathrm{IH}_{9,17}$.

Similar to the previous, set $D$ is not the minimum dominance set for network $\mathrm{IH}_{9,17}$.

Node $R_{21}^{1} \equiv R_{21}^{5}$ can be removed because it dominates over nodes dominated by nodes $R_{12}^{1}, R_{41}^{1}$, and $R_{21}^{5}$. Also, node $R_{21}^{4} \equiv R_{21}^{3}$ can be removed because it dominates over nodes dominated by $R_{12}^{4}, R_{41}^{4}$, and $R_{21}^{3}$.

Let us consider nodes in set $D$ that satisfy the condition that their mutual distance is equal to 1 and the assumption that one of them is removed from set $D$. If we throw out node $R_{42}^{2}$, node $R_{33}^{2}$ would remain without dominance. If we dropped the node $R_{41}^{5} \equiv R_{41}^{4}$, the node $R_{24}^{4}$ would remain without dominance. The same is true for the other nodes in the set $D$. Based on that, the dominating set for the network $\mathrm{IH}_{9,17}$ is the set $D^{\prime}=D /\left\{R_{21}^{1}, R_{21}^{4}\right\}$.
Its cardinality is $5 \cdot 32-2$, i.e., 158 . Hence, $\gamma\left(\mathrm{IH}_{9,17}\right)=$ 158 which had to be proved.

Theorem 4. For $n=2^{k} i k \in N /\{1\}$, the dominating number $\gamma$ $\left(\mathrm{IH}_{n+1,2 n+1}\right)=5 \cdot 2^{2 k-1-} 2$.

Proof. Based on the results proved in Lemma 4, the dominance number for the rhomboidal-triangular network $\gamma\left(R_{n+1}, 2 n+1\right)=2^{2 k-1}$. The icosahedral-hexagonal network is composed of 5 rhomboidal-triangular networks that have a common start $R_{1}$, where node $R_{1}$ does not belong to the dominating set of the rhomboidal-triangular network. Based on that, we conclude that the dominating number of the icosahedral-hexagonal network is $\gamma\left(\mathrm{IH}_{n+1,2 n+1}\right) \leq 5 \cdot \gamma$ $\left(R_{n+1}, 2 n+1\right)$. The dominance set on the icosahedral-hexagonal network $\mathrm{IH}_{n+1,2 n+1}$ is denoted by $D$. Since there are 
5 nodes of the dominating set $D$ at the distance 1 from the common beginning $R_{1}$, we will consider the possibility of removing some of these 5 nodes from the set $D$. Analogous to the proof of Theorem 3, we conclude that, from set $D$ it is possible to remove only 2 nodes among the 5 nodes that form the first circle around the central node $R_{1}$. In addition to the common node $R_{1}$, the rhomboidal-triangular networks in the icosahedral-hexagonal network touch parts of their edges. By removing any node from the tangent edges as well as any other node from the set $D$, at least one node in the network $\mathrm{IH}_{n+1,2 n+1}$ would remain without dominance. Based on that, we conclude that it is $\gamma\left(\mathrm{IH}_{n+1,2 n+1}\right)=5 \cdot \gamma$ $\left(R_{n+1,2 n+1}\right)-2$. Thus, based on Lemma 4 , we obtain $\gamma$ $\left(\mathrm{IH}_{n+1,2 n+1}\right)=5 \cdot 2^{2 k-1}-2$ which needed to be proven.

\section{Conclusion}

Starting from the rhomboidal-triangular network $R_{2,3}$ which is composed of 2 rhombuses, i.e., 4 triangles from the icosahedral-hexagonal network for which the dominance number is determined in [3], we determined the dominance number for the rhomboidal-triangular network $R_{m, c}$ composed of $m$ rows and $c$ columns. The network $R_{m, c}$ is formed by dividing the edges of the triangles in the initial network $R_{2,3}$ into $n=2^{k}$ parts where $k \in N \backslash\{1\}$. We have proved that the dominating number is $\gamma\left(R_{n+1,2 n+1}\right)=2^{2 k-1}$.

By dividing the edges of the triangles in the icosahedralhexagonal network $\mathrm{IH}_{2,3}$ by $n=2^{k}$ equal parts, we obtained the icosahedral-hexagonal network $\mathrm{IH}_{n+1,2 n+1}$. We determined the dominance number for the icosahedral-hexagonal network $\mathrm{IH}_{3,5}$ and then for $\mathrm{IH}_{n+1,2 n+1}$ where $n=2^{k}$ and $k \in N /\{1\}$ as in the rhomboidal-triangular network. We proved that $\gamma\left(\mathrm{IH}_{n+1,2 n+1}\right)=5 \cdot 2^{2 k-1-} 2$.

In this paper, we determined the 1-dominating sets and the 1-domination numbers on an icosahedral-hexagonal network. In future works, we plan to explore and determine $t$-domination on the icosahedral-hexagonal grid for $t>1$.

\section{Data Availability}

Guides to the data that support the conclusions of the study are given in the sections of this paper.

\section{Conflicts of Interest}

The author declares that there are no conflicts of interest regarding the publication of this paper.

\section{References}

[1] D. Majewski, D. Liermann, P. Prohl et al., "The operational global icosahedral-hexagonal gridpoint model GME: description and high-resolution tests," Monthly Weather Review, vol. 130, no. 2, pp. 319-338, 2002.

[2] M. Mihajlov Carević, M. J. Petrović, and N. Denić, "Generating function for the figurative numbers of regular polyhedron," Mathematical Problems in Engineering, vol. 2020, Article ID 6238934, 7 pages, 2020.

[3] M. M. Carević, M. Petrović, and N. Denić, "Dominating sets on the rhomboidal cactus chains and the icosahedral network," in Proceedings of the 19th International Symposium
INFOTEH-JAHORINA, Collection of Papers, pp. 152-157, Dublin, Ireland, April 2020.

[4] E. J. Farrell, "Matchings in hexagonal cacti," International Journal of Mathematics and Mathematical Sciences, vol. 10, no. 2, pp. 321-338, 1987.

[5] T. Došlić and F. Måløy, "Chain hexagonal cacti: matchings and independent sets," Discrete Mathematics, vol. 310, no. 12, pp. 1676-1690, 2010.

[6] Z. Yarahmadi, T. Došlić, and S. Moradi, "Chain hexagonal cacti: extremal with respect to the eccentric connectivity index," Iranian Journal of Mathematical Chemistry, vol. 4, no. 1, pp. 123-136, 2013.

[7] S. Majstorovic, T. Doslic, and A. Klobucar, "k-Domination on hexagonal cactus chains," Kragujevac Journal of Mathematics, vol. 36, no. 2, pp. 335-347, 2012.

[8] E. J. Farrell, "Matchings in rectangular cacti," Mathematical Science (Calcutta), vol. 9, pp. 163-183, 1998.

[9] E. J. Farrell, "Matchings in triangular cacti," Mathematical Science (Calcutta), vol. 11, pp. 85-98, 2000.

[10] P. J. Slater, "R-domination in graphs," Journal of the ACM, vol. 23, no. 3, pp. 446-450, 1976.

[11] A. Klobučar, "On the \$ k \$-dominating number of Cartesian products of two paths," Mathematica Slovaca, vol. 55, no. 2, pp. 141-154, 2005.

[12] T. W. Haynes and P. J. Slater, "Paired-domination in graphs," Networks, vol. 32, no. 3, pp. 199-206, 1998.

[13] A. Klobučar and A. Klobučar, "Total and double total domination number on hexagonal grid," Mathematics, vol. 7, no. 11, p. 1110, 2019. 